\title{
Tecnologías de la Información y Comunicación exclusivo para el comportamiento del consumidor desde una perspectiva teórica
}

\section{Information and Communication Technologies exclusively for consumer behavior from a theoretical perspective}

\begin{abstract}
Mg. Freddy Rodolfo Lalaleo-Analuisa es gerente general de la Editorial Queyám Cía. Ltda. (Ecuador)
\end{abstract} (flalaleo@indoamerica.edu.ec) (https://orcid.org/0000-0003-0108-3365)

Mg. Diego Mauricio Bonilla-Jurado es investigador asociado de la Universidad Tecnológica Indoamérica-Matriz Ambato (Ecuador) (diegobonilla@uti.edu.ec) (https://orcid.org/0000-0002-4784-7861)

Mg. Rodolfo Enrique Robles-Salguero es docente investigador de la Universidad Estatal de Milagro (Ecuador) (rrobless@unemi.edu.ec) (https://orcid.org/0000-0003-1016-4079)

\section{Resumen}

El objetivo de este estudio se centra en realizar una revisión de literatura de las Tecnologías de la Información y Comunicación (TIC) como un factor exclusivo que incide en el comportamiento del consumidor; la metodología tiene un alcance descriptivo de tipo bibliográfico, se seleccionaron estudios que han sido publicados en los índices más importantes a nivel mundial, en el siguiente orden: Web of Science, Scopus, Ebsco y ProQuest, desde el año 2009 hasta el 2020, con un total de 62 publicaciones entre artículos científicos, libros y tesis, que discutieron el desarrollo de las TIC exclusivamente enfocado hacia el consumidor moderno. Se encontró una considerable producción científica entre los años 2013 y 2017 con un $47,00 \%$ de los documentos analizados. Asimismo, en dichas publicaciones se resaltan palabras clave como TIC, consumidores digitales y comportamiento de consumidor con el 27,00\%, 19,00\% y 10,00\% respectivamente, en cuanto a la procedencia de los documentos se destaca que la mayoría de estos provienen de América Latina con un porcentaje del 53,00\%. Como principales conclusiones se destaca que la intervención de las TIC está inmersa en casi todas las fases de la toma de decisiones que realiza el consumidor, adicional a ello, los consumidores actuales se encuentran permanentemente conectados, disponiendo mayores alternativas de elección según la información que la empresa desarrolla para alcanzar la compra final del producto y/o servicio.

\begin{abstract}
The objective of this study, focuses on carrying out a literature review of Information and Communication Technologies (ICT) as an exclusive factor affecting consumer behavior. The methodology has a descriptive scope of a bibliographic type, studies were selected that have been published in the most important indexes worldwide, in the following order: Web of Science, Scopus, Ebsco and ProQuest, from 2009 to 2020, with a total of 62 publications among scientific articles, books and thesis, which discussed the development of ICT exclusively focused on the modern consumer. A considerable scientific production found between 2013 and 2017 with 47,00\% of the documents analyzed. Likewise, in these publications key words such as ICT, digital consumers and consumer behavior are highlighted with 27,00\%, 19,00\% and 10,00\% respectively. Regarding the origin of the documents, it is highlighted that most of them come from Latin America with a percentage of 53,00\%. As main conclusions it is highlighted that the intervention of the TIC is immersed in almost all the phases of the decision making that the consumer makes, in addition to it, the present consumers are permanently connected, having greater alternatives of election according to the information that the company develops to reach the final purchase of the product and/or service.
\end{abstract}

\section{Palabras clave I keywords}

Comercio electrónico, comportamiento del consumidor, consumidores digitales, e-commerce, entorno digital, internet, social media, Tecnologías de la Información y Comunicación (TIC).

E-commerce, consumer behavior, digital consumers, digital environment, internet, social media, Information and Communication Technologies (ICT).

Cómo citar: Lalaleo-Analuisa, F.R., Bonilla-Jurado, D.M., y Robles-Salguero, R.E. (2021). Tecnologías de la Información y Comunicación exclusivo para el comportamiento del consumidor desde una perspectiva teórica. Retos Revista de Ciencias de la Administración y Economía,11(21), pp. 147164. https://doi.org/10.17163/ret.n21.2021.09. 


\section{Introducción}

El impacto que ha tenido el Internet en los últimos años ha propiciado que cambie en gran medida el comportamiento del consumidor. Hoy en día, tanto empresas como consumidores sacan provecho a todas las bondades que generan las operaciones de compra y venta por este medio; una buena cantidad de consumidores en función de los nuevos esquemas culturales y sociales surgidos con ocasión de cambio del estilo de vida, encuentran una buena opción para adquirir prácticamente cualquier producto y/o servicio para satisfacer sus distintas necesidades a través de las nuevas tecnologías de información. En este sentido, el avance tecnológico registrado permitió que las Tecnologías de la Información y Comunicación en adelante (TIC) jueguen un papel importante especialmente durante el último año, donde a consecuencia de la COVID-19, el planeta experimentó un cambio radical en relación al desarrollo de las diferentes actividades.

El aporte de este estudio es generar conocimiento para que las organizaciones comprendan la conducta del consumidor que se está formando por el desarrollo de las TIC, donde a través de la revisión de literatura se identificó elementos y factores que debe tomar en cuenta la alta gerencia de la empresa, y al mismo tiempo dar respuesta a muchas interrogantes que se hacen los empresarios sobre ¿quiénes son los consumidores? ¿cuáles son sus características demográficas y psicográficas? ¿qué información es relevante para atraer a estos consumidores? ¿qué compran? ¿cada cuánto compran? ¿por qué medios compran? ¿a todos les atrae lo mismo?, y todas las preguntas que influyen en la investigación de mercados. Adicional a ello, las conclusiones de este estudio generan valor en la información, sobre la cual los productos y/o servicios deben diseñarse con estrategias específicas en el comportamiento del consumidor para maximizar las ventas a través de las TIC, y al mismo tiempo identificar grupos de referencia con los cuales se aprovechan las oportunidades que aparecen de forma indistinta.

Las empresas del siglo XXI continúan transformándose debido a la globalización del internet y con ello, el vertiginoso crecimiento de las redes sociales y las diferentes aplicaciones móviles, acontecimiento que ha generado distintos cambios en los ámbitos internos como externos de las organizaciones. En este sentido, estas necesariamente deben adaptarse a escenarios que cada día son más competitivos y complejos, donde los administradores deben poseer la suficiente habilidad, ingenio y experticia para diseñar estrategias de valor para llegar a satisfacer a un nuevo comportamiento y estilo de vida de los diferentes demandantes de productos y/o servicios que influenciados en gran medida por aquellas publicaciones presentadas a través de los distintos medios virtuales se encuentran cada vez más seducidos (Aucay \& Herrera, 2017).

Hoy, en plena era de la información o como otros teóricos lo denominan la era de la revolución de las TIC (Restrepo, 2011), se encuentran alterando rápidamente el modo en que las personas hacen negocios en todo el mundo. En el segmento de negocio a consumidor, las ventas a través de la web han aumentado radicalmente durante los últimos años. Dado que, los clientes, no solo de aquellos países desarrollados sino también de países en vías de desarrollo, están adoptando un nuevo canal de compras (Barrientos, 2017). En tal virtud, las TIC tienen un papel importante que desempeñar no solo en las empresas, sino también en las actividades diarias de las personas. Los servicios con base en internet siguen creciendo en el entorno de "empresa a consumidor" y "empresa a empresa" (OCDE, 2019). Desde este punto de vista, cada año, el número de organizaciones que usan internet con fines de marketing se incrementa en gran medida. Así como también, el número de consumidores que utilizan internet no solo para recopilar información sino también para realizar la compra de bienes (Rivera, 2015). 
En un contexto general, se estima una población total de 7676000000 de personas de las cuales el 75,00\% están considerados como usuarios activos de internet (Arribas et al., 2019). Mientras que, en relación con el número de usuarios activos en redes sociales se estima una cantidad cercana a los 3484000000 que corresponde al $45,00 \%$ de la población global, siendo Facebook, la red social con mayor número de suscritores (Kutchera et al., 2014), seguido por YouTube y WhatsApp respectivamente. Por su parte, en el continente americano, específicamente en el área de América del Sur, se estima una población general cercana a los 430000000 de personas de las cuales el 73,00\% son usuarios permanentes de internet; es decir, en comparación con el porcentaje a nivel mundial este indicador aumenta significativamente en esta región (Islas et al., 2018) (Ver Figura 1).

\section{Figura 1. Uso y conectividad en el mundo}

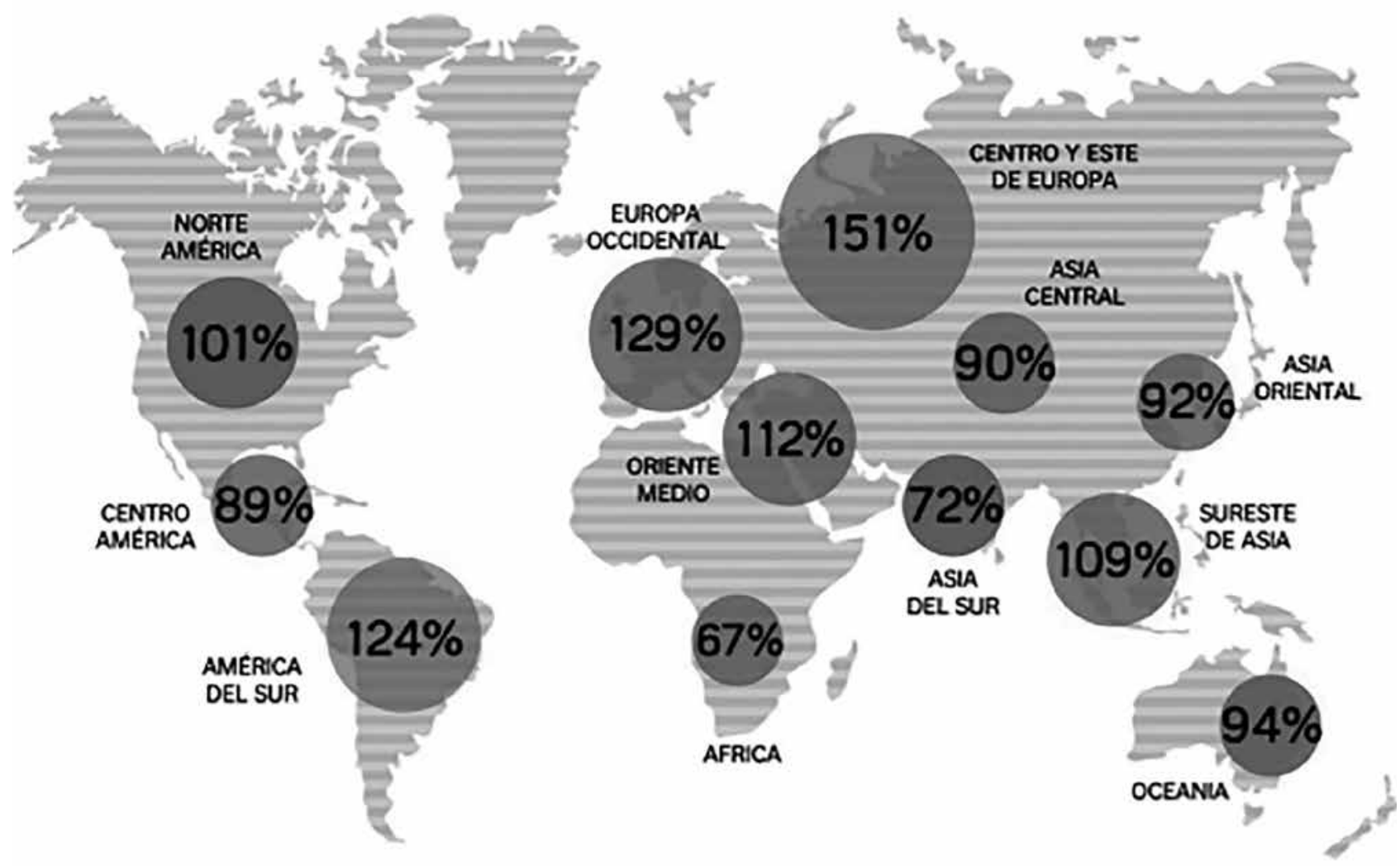

Fuente: Adaptado de Campos, 2015.

Con estas importantes estadísticas, hoy en día, en el contexto mundial es muy común ver o escuchar que la mayoría de las ventas se efectúan vía online; es decir, un tipo de comercio en donde se utiliza como principal medio para realizar dichas transacciones un sitio web o una aplicación conectada a internet (Roman, 2016). En la última década, la práctica de compra de los clientes ha variado de forma radical, pues el uso de la tecnología digital para navegar, investigar y comprar ha pasado de un segmento esporádico o específico a la corriente principal. Ante esta realidad, diferentes estudios revelan información sobre cómo los clientes utilizan la tecnología y sus expectativas sobre las empresas en este entorno nuevo (Cano, 2018; Encalada et al., 2019; Linero \& Botero, 2020; Matute et al., 2012; Millat, 2011; Vera et al., 2011). Sobre este razonamiento, actualmente distintas organizaciones se ven obligadas a plantear y utilizar 
nuevas estrategias que involucran principalmente el uso de la tecnología como factor indispensable en sus empresas, para dar respuesta oportuna tanto a las necesidades como a las expectativas de sus clientes. Es decir, a medida que estas brinden innovaciones para mejorar la experiencia de compra, las expectativas de los clientes cambiarán, es por ello que, en la actualidad la inversión en tecnología digital se está convirtiendo en un imperativo estratégico significativo para el afianzamiento y desarrollo de muchas empresas a escala mundial (Yasav, 2015).

La sociedad actual se halla expuesta a continuos cambios en el ámbito tecnológico, donde el aspecto digital toma una ventaja significativa y las personas constituyen el punto céntrico del desarrollo digital experimentado, es así que, las prácticas de la vida digital van de la mano con la evolución tecnológica (Santos, 2019). En este sentido, la evolución de las TIC ha traído consigo una serie de mecanismos y herramientas de interconectividad e intermediación (Díaz et al., 2011; Madrigal et al., 2015). El mundo de hoy se encuentra conquistado por una gran cantidad de aparatos móviles que están inmersos en cada una de las actividades diarias que realizan las personas, dispositivos tecnológicos que son utilizados por éstos con la finalidad de informarse, analizar la realidad, activar relaciones con otros y principalmente comunicarse (Ureña et al., 2014). Este escenario ha traído consigo principalmente el reajuste en relación con las formas de comunicación entre pares, al tiempo que se ha encargado de imponer una escala nueva de valores y canales de intercambio cuya característica principal está dada por la existencia de una mayor interacción en la relación empresa/cliente (Barrios, 2014).

En el ámbito comercial, la utilización de las TIC conduce a un mayor número de mercados pudiendo ser estos locales, nacionales e internacionales, al mismo tiempo que contribuyen a una mayor transparencia en relación con la información, permitiendo de esta manera la racionalización de los costos operativos, así como también precios más bajos (Saavedra \& Tapia, 2013). Desde este punto de vista, su aplicación facilita el acceso a las cadenas de suministro mundiales donde tanto compradores como vendedores comparten información, especificaciones y procesos, los cuales traspasan fronteras. Es así que, tanto la red de compradores y vendedores como las distintas herramientas de investigación proporcionan de manera electrónica una evaluación rápida, apropiada y económica de la oferta entre diferentes proveedores, con una realización y finalización eficiente de todas las transacciones implícitas en el proceso de promoción y adquisición (Mihajlović et al., 2014).

Asimismo, puede mencionarse que el comportamiento actual que presenta el cliente afecta de sobremanera a todos los involucrados en la cadena de comercialización quienes se enfocan en la adquisición ya sea de bienes o servicios, los cuales son destinados a satisfacer las necesidades existentes (Virgüez et al., 2020). Dado que, con el paso de los años, atrás quedó la producción básica en la cual, los individuos únicamente producían lo justo y necesario que les permitía su subsistencia a una producción en masa en la actualidad, la misma que permite atender a un mercado altamente competitivo en donde la publicidad de alto impacto es la que mayormente llama la atención de los clientes (Díaz \& Vicente, 2011).

Por su parte, los clientes que han incursionado en el ámbito digital han desarrollado prácticas nuevas de consumo, situación que se genera debido al constante avance de las TIC, esta evolución ha dado lugar a la aparición de nuevas aplicaciones que han ocasionado un desarrollo constante en los productos (Guaña et al., 2015). No obstante, a pesar de ello, no todo resulta bueno, pues este constante desarrollo trae consigo un grave problema asociado especialmente a cómo comprender las necesidades del denominado "nuevo consumidor", dado que, este por naturaleza cambia su accionar de forma inesperada; cambio que puede ser atribuido debido a que en la actualidad, "el usuario se halla conectado a internet las 24 horas del día, los 7 días de la semana y 
los 365 días del año" (Lagos et al., 2018). Esto sin duda alguna ha creado una perturbación constante en el usuario, el mismo que se encuentra expuesto a una infinidad de información, productos y/o servicios y promociones (Guaña et al., 2017).

Ante este escenario, estos nuevos consumidores, denominados clientes digitales suelen utilizar diferentes herramientas asociadas al entorno digital como una vía de información compartida, lo que permite a las nuevas tecnologías desempeñar un papel importante en la vida de cada uno de los individuos (Apolo et al., 2015). En un contexto general, este tipo de clientes aumentan de manera considerable, aumento que se asocia principalmente con los distintos beneficios que otorga el uso del internet, razón por la cual, las organizaciones se ven obligadas a modificar su estructura de negocio, así como también necesariamente los clientes tienen que acoplarse a los nuevos procesos que se realizan de manera efectiva, estableciendo al comercio electrónico como una alternativa fundamental para el crecimiento sostenible de las diferentes organizaciones.

En este mismo orden de cosas, aparece el término consumidor 2.0 que puede ser definido como:

Una persona bien informada, que se aburre rápidamente y es bastante inmune a la publicidad obvia, busca información innovadora y experiencias diferentes. Sobre esa base, los nuevos consumidores están conectados permanentemente, tienen mayor poder de decisión, más posibilidad de elección y son más demandantes de información que nunca. (Cáceres et al., 2017, p. 15)

Bajo los parámetros señalados, un consumidor 2.0 constituye el actual público meta para cualquier tipo de organización; es decir, si se logra captar su interés y más aún su consumo existen grandes probabilidades de tener éxito en un mercado cada día más competitivo y lleno de marcas que comercializan una infinidad de productos y/o servicios. En síntesis, aprovecha las posibilidades que le ofrece la web para mejorar su experiencia de compra (Burgos et al., 2009). Al contrario que el consumidor tradicional, dispone de una variedad inmensa de recursos y herramientas que le permiten tener una posición aventajada al realizar compras, ya sea online u offline. Adicional a lo señalado, es necesario recalcar que, este no realiza simplemente una compra, al contrario reclama una experiencia positiva en el proceso de compra, reclama información, transparencia, comunicación, asesoramiento, atención personalizada, contenidos prácticos, así como también calidad a un bajo costo (Ferrer, 2018).

Con las exigencias enunciadas en el párrafo anterior, el desarrollo de las TIC apoya la interacción entre los consumidores y la intermediación, como resultado, conduce a la reingeniería del negocio, el desarrollo de procesos, así como las formas de comunicación (López et al., 2018). Adicional a ello, las tecnologías innovadoras mejoran la eficiencia para clientes y proveedores, además fomentan la interoperabilidad, la personalización y la creación de redes permanentes de los participantes en el proceso (Travaglini, 2016). En la actualidad, las TIC han ganado un protagonismo significativo en diferentes actividades como el ámbito educativo, el sector de la salud, y la economía en general. Dado que, durante los últimos años su inclusión en la vida diaria de las personas es innegable, pues su uso está inmerso en las distintas actividades que son desarrolladas día tras día (Guaña et al., 2017).

Las TIC representan una de las principales fuerzas impulsoras detrás de los cambios económicos, sociales y culturales de lo moderno a lo posmoderno, de los mercados locales a los globales, de la producción al consumo y de las economías industriales a las informativas. Con la disponibilidad de las TIC prácticamente en todo el mundo, las experiencias de consumo y el marketing se encuentran cada vez más representados a través del concepto de datos. Es decir, la información, hoy más que nunca, se ha trans- 
formado en la parte esencial del mercado (Ferreirós, 2016). Los consumidores a menudo se sienten abrumados e intimidados por el gran volumen de información disponible para el consumo (Rivera, 2015). No obstante, y como respuesta a dicha sobrecarga de información, los especialistas en marketing han respondido con posibles soluciones que de cierta manera parecieran imitar al problema planteado; a la avalancha de información disponible existe la misma cantidad de herramientas de búsqueda acompañadas con guías de compra, donde se ofrece resúmenes altamente específicos para mayor comodidad de quienes lo utilicen.

Por otro lado, desde el punto de vista del marketing y el consumo, el impacto de la alta tecnología y las TIC ha sido la transformación de los espacios de mercado físicos en paisajes de datos que abarcan no solo un porcentaje creciente de transacciones de clientes sino también, por ejemplo, el seguimiento en tiempo real del movimiento global de la mercadería (Capellan et al., 2015). Los procesos de adopción de tecnología poco a poco han llamado la atención de los estudiosos en marketing y ciencias sociales durante varias décadas atrás, dado que, la tecnología ha sido reconocida como un factor clave que impulsa la estrategia, no únicamente en los aspectos de investigación y desarrollo (I+D), sino también las carteras de marketing y productos o servicios de muchas organizaciones (Dholakia et al., 2009).

Por lo general, las organizaciones emplean las TIC para una comunicación interna y externa, para la actualización e intercambio fácil y rápido de información y, para aumentar su competitividad a través de la creación de webs, portales, foros de discusión, tiendas electrónicas, entre otros (Gaile, 2009).

En este mismo orden de cosas, las TIC en poco tiempo se han convertido en un factor clave para la obtención de una importante ventaja competitiva en las organizaciones. Es así que, la popularización en relación con su uso ha generado una transformación evidente en un número considerable de áreas de las diferentes empresas, pues con su aplicación, hoy en día resulta más fácil realizar un procesamiento de pedidos, una gestión efectiva de existencias y obviamente el almacenamiento y transporte si se trata de una empresa de producción. Adicional a lo señalado, con la aplicación correcta de las TIC en el contexto empresarial se pueden desprender varios beneficios adicionales para una organización como es el caso del ahorro de tiempo, gastos innecesarios en el personal, mayor productividad, mejor gestión de inventarios, disminución de errores, exactitud y fiabilidad (Gil et al., 2009).

Hoy en día, a través del uso de las TIC, los clientes tienen mayor acceso a la información que antes (Barinas, 2013). Es decir, buscan en la web información relacionada a los bienes y/o servicios que necesitan para complacer sus necesidades existentes. Este proceso de sofisticación del cliente obliga a los administradores de negocios a innovar con mayor precisión y rapidez en las soluciones que ofrecen sus empresas ante los requerimientos de los usuarios. En tal virtud, afortunadamente, la información que es capturada mediante las TIC puede ayudar a los distintos administradores en el duro proceso de comprender el comportamiento de los consumidores y las soluciones disponibles en el mercado (Barrio, 2017).

Por consiguiente, la información de los perfiles de las personas que indagan en la web, sobre lo que se encuentran buscando, los canales utilizados y el tipo de dispositivo utilizado para realizar dichas consultas, es ofrecido por grandes organizaciones como Google y Facebook, las cuales se enfocan en brindar información referente al cliente y ayuda también a las diferentes empresas a ser visibles para los clientes reales y potenciales. De forma particular, Google Analytics se encarga de ofrecer soluciones para comprender la interacción de los clientes con distintos canales de marketing en línea. Por ejemplo, cuenta con herramientas especializadas que brindan información útil 
para reconocer cómo los clientes utilizan las tecnologías móviles y medir el éxito de las campañas de redes sociales. Asimismo, un servicio más que proporciona datos y ofrece herramientas para analizar lo que las personas buscan en la web; desarrollar proyectos de investigación de mercado y comprender las tendencias del sector es Google Insight (Piñeros \& Gómez, 2017). Por su parte, Facebook constituye otra organización que brinda información de los clientes; dado que, esta compañía creó una de las redes sociales con mayor tráfico de personas en el planeta, ofreciendo servicios de marketing para empresas de distintos segmentos (Blanes, 2017). Al existir una gran cantidad de datos, esta empresa se encarga del análisis de los mismos con la finalidad de diseñar campañas publicitarias para conjuntos específicos de clientes potenciales que logran un alto impacto en el ámbito publicitario. En este sentido, la calidad de la información obtenida permite localizar geográficamente a los clientes y ofrecer una interpretación integral de las necesidades e intereses de los clientes.

En definitiva, tanto Google como Facebook utilizan la información generada a través del uso de las TIC, para ayudar a los administradores y sus equipos a tomar decisiones más precisas. No obstante, para realizar un análisis más confiable y, en consecuencia, tomar decisiones más racionales, las empresas pueden utilizar la información registrada en su contacto diario con clientes reales y potenciales (Piñeros \& Gómez, 2017). La adopción generalizada de las TIC, así como su uso continuo, independientemente del tiempo y el lugar, refuerza el auge del comercio digital (Rivera et al., 2012). Por consiguiente, esta forma emergente de realizar transacciones comerciales se refiere al intercambio de valor unidireccional o bidireccional facilitado por un dispositivo electrónico, el mismo que está habilitado por tecnologías inalámbricas y redes de comunicación (Golovina, 2014). La toma de decisiones del consumidor ha cambiado drásticamente desde la prevalencia de estos dispositivos en la vida cotidiana del consumidor (Pelet \& Papadopoulou, 2013). Con fácil acceso a reseñas de usuarios, opiniones de expertos, comparaciones de precios y otras instalaciones emergentes, los consumidores pueden realizar evaluaciones exhaustivas de los productos y servicios disponibles en un número cada vez mayor de categorías. Para los especialistas en marketing, esto sugiere la necesidad de una forma totalmente nueva de pensar sobre cómo influir en los consumidores. El desafío también es válido para los desarrolladores de sistemas de información, ya que los sitios web de comercio móvil y las redes sociales deben implementarse y utilizarse cuidadosamente para convertirse en herramientas de marketing eficaces (Pelet, 2014).

Independientemente de los medios de comunicación que se utilice, la relación de estos con el cliente se da de forma bidireccional; es decir, en primera instancia, los medios tienen gran injerencia en el comportamiento del consumidor debido a que genera en ellos ciertos efectos de tipo afectivo, cognitivo y actitudinal (Vega, 2015); mientras que, la otra vía determina que los clientes ya no son considerados únicamente como actores pasivos de la información, sino que pasan a ser usuarios activos donde se convierten en expertos comunicadores, pues se encargan de difundir información, realizar valoraciones e inclusive emitir comentarios personales (Golovina, 2014).

Asimismo, el entorno dinámico actual que conjuga la era digital y el cambiante contexto económico, obliga a las diferentes compañías a investigar sobre las preferencias de los consumidores, realizando estudios de mercados y conociendo las actividades que ejecutan las empresas competidoras con la finalidad de tener un panorama real que permita lanzar al mercado un producto y/o servicio que brinde importantes réditos económicos. Sobre esa base, la información disponible del mercado ayuda a la parte administrativa y de marketing en la toma de decisiones; dado que, el enfoque no se limita únicamente a reunir datos, sino que busca la manera más idónea para some- 
terlos a un análisis exhaustivo con la finalidad de identificar tendencias y datos estadísticos que permitan delinear acciones oportunas dentro de la gestión organizacional (Ortiz et al., 2016). Es por ello que, a continuación, se hace un análisis descriptivo a través de una revisión de literatura en torno a las TIC y el comportamiento del consumidor, partiendo desde los antecedentes anteriormente establecidos.

\section{Materiales y método}

Con la finalidad de comprobar el interés en el ámbito científico académico sobre la temática planteada (TIC y comportamiento del consumidor) durante los últimos años, se desarrolla un estudio de tipo bibliográfico, el mismo que se enfocó en la revisión de literatura de distintas fuentes de información de un nivel secundario (artículos científicos, libros, investigaciones realizadas con anterioridad). Para ello, se han analizado documentos científicos que han sido publicados y cuyos textos se pueden recuperar de bases de datos como Scopus, ProQuest, Ebsco, Web of Science.

Con base en lo expuesto, seguidamente se plantea los múltiples propósitos que implica una revisión de la literatura pues constituye una de las partes más importantes de la génesis de un estudio. Esta perpectiva permite al investigador conocer resultados de otros estudios similares al que se está planteando, además provee de un marco de referencia para justificar la importancia del estudio, asimismo, permite tener un punto de referencia para comparar los resultados obtenidos con otros que se han derivado de anteriores investigaciones dentro de un contexto similar al establecido (Creswell, 2014). Sobre esa base, científicamente está demostrado que los artículos de revisión se encargan de compactar y sintetizar los conocimientos fragmentados, actualizar e informar sobre el estado de un tema particular, conocer la tendencia de las investigaciones, comparar la información de distintas fuentes, evaluar la literatura publicada, entre otras (Cué et al., 2008).

Bajo este contexto y viendo la importancia de una revisión bibliográfica, en primera instancia se tomó en consideración aquellos trabajos que han sido difundidos durante la última década, ya sea a través de una presentación física o mediante el uso de plataformas electrónicas como revistas indexadas o repositorios digitales. En relación con los vocablos que posibilitaron la búsqueda se utilizaron las palabras o acrónimos clave como lo destacan Bonilla et al. (2019) siendo estos por ejemplo: TIC, comportamiento del consumidor, consumidor 2.0, era digital, internet, marketing digital. Estos términos resaltados constituyen la base para la búsqueda correspondiente de información en relación con el tema planteado. A partir de lo señalado, para la indagación de información fue necesario delimitar los años de búsqueda (2009-2020) de forma que no se presente información muy antigua que pudiera distorsionar el contexto actual de la temática establecida. Adicional a ello, resulta necesario aclarar que la elección de estos años se debe principalmente a los avances registrados en torno a las TIC durante aquel período. Finalmente, en cuanto se refiere al idioma de publicación no existe restricción alguna para la selección de los documentos. Sobre esta base, los trabajos que se ajustaban a los requerimientos establecidos y de donde se obtuvo la información fueron de un total de 62 fuentes confiables y con una calidad de información.

Adicional a lo señalado, el procesamiento de la información obtenida tras la búsqueda ejecutada se efectuó de la siguiente manera: 
Figura 2. Procesamiento de la información

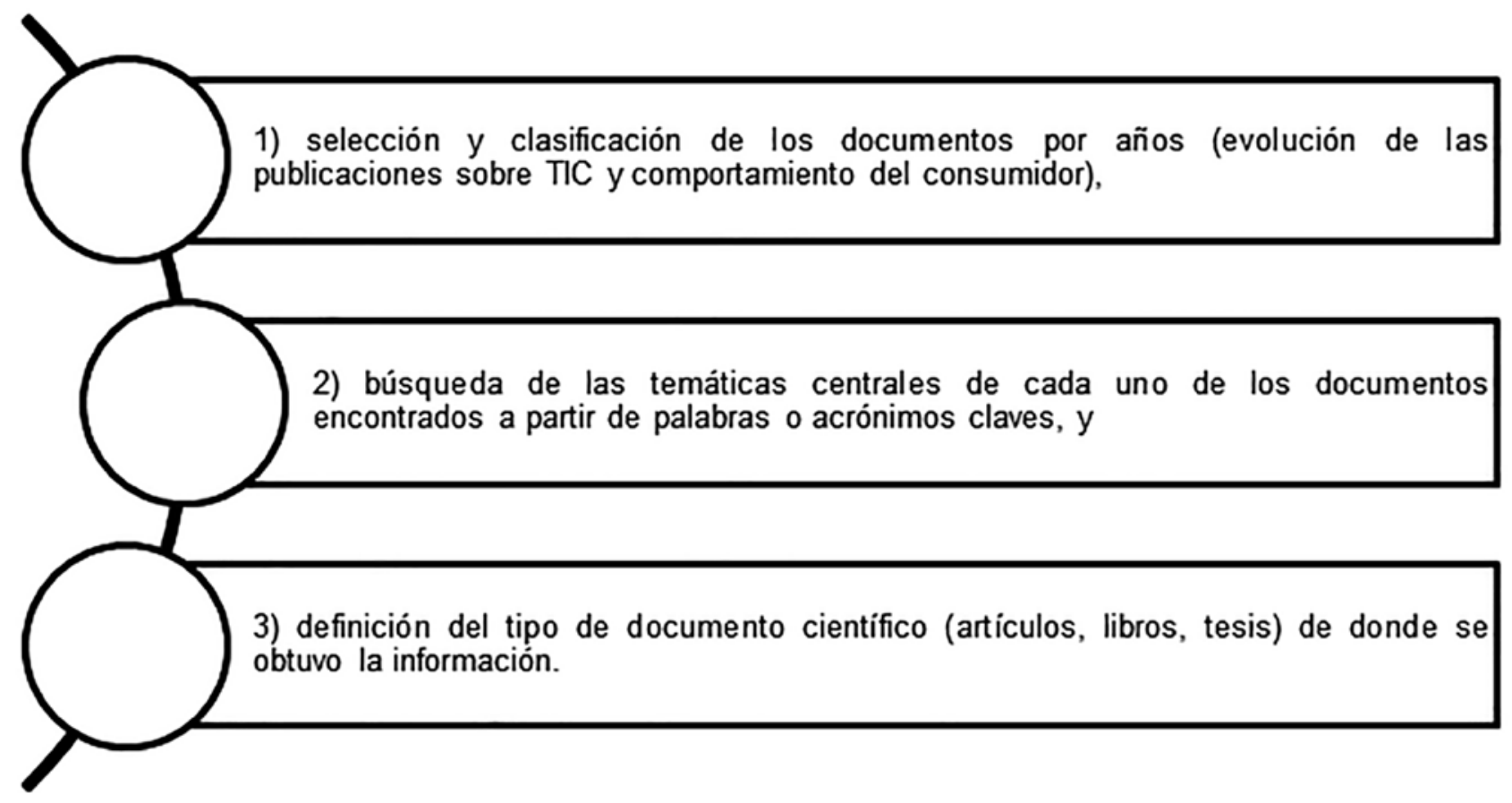

Fuente: Elaboración propia.

Finalmente, los análisis se han centrado en agrupar los distintos elementos de esta unidad muestral de fuentes, que se esperan sean representativas por su forma de selección, en categorías que agrupen los estudios en las más relevantes clasificaciones para evaluar la producción científica. De esta manera, se ha categorizado la producción relativa a los años, las áreas temáticas en las que se han centrado los estudios, el tipo de documento científico y los países de publicación, este procedimiento realizado fue similar al presentado dentro del estudio desarrollado por Sánchez et al. (2019).

\section{Resultados}

Dentro de este apartado, se inicia el mismo presentando la producción científica (documentos) por años en torno al tema planteado, dentro del análisis temporal se delimitó aquellas publicaciones realizadas entre el año 2009 y 2020, dado que, es evidente el desarrollo que ha experimentado las TIC durante este periodo de tiempo y cómo ello ha influido en el modus vivendi de las diferentes personas. 


\section{Figura 3. Evolución de las publicaciones sobre TIC y comportamiento del consumidor}

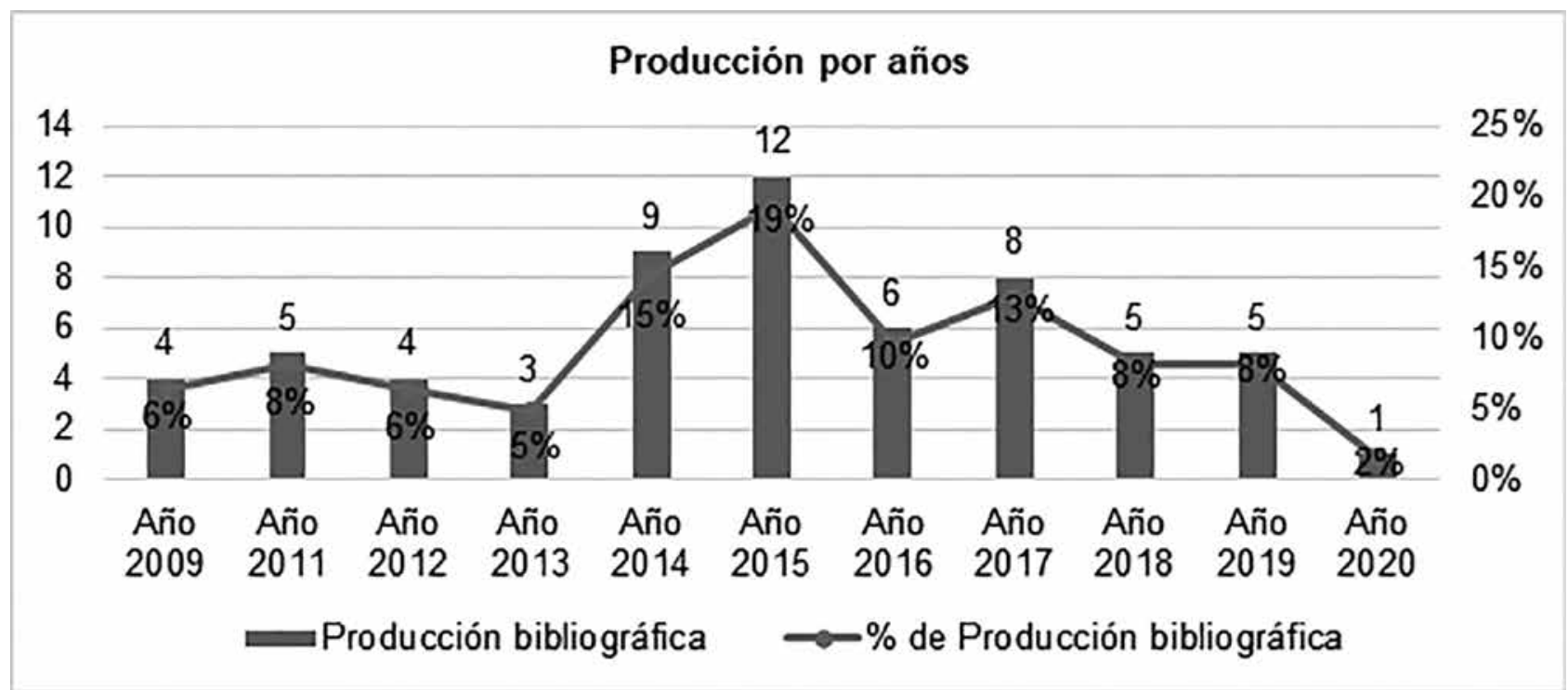

Fuente: Elaboración propia.

En términos porcentuales, los años que presentan mayor producción científica en este campo fueron 2014, 2015 y 2017 con porcentajes del 15,00\%, 19,00\% y 13,00\% respectivamente, mientras que, el 2013 según las estadísticas planteadas sería el año con menos producción científica con apenas el 5,00\% del total de la producción. Asimismo, resulta necesario hacer una mención especial al año 2020 que si bien es cierto presenta una baja producción científica no podría ser considerado puesto que los registros obtenidos únicamente constituyen aquella producción alcanzada durante el primer trimestre del presente año.

Por otro lado, en relación con las áreas temáticas que aborda la producción científica analizada, aquellas que más se han estudiado dentro de estos últimos años son las TIC $(27,00 \%)$ y consumidores digitales $(19,00 \%)$. Seguidamente, se destaca las áreas como: comportamiento del consumidor $(10,00 \%)$, marketing digital $(6,00 \%)$, entorno digital $(6,00 \%)$, e-commerce $(5,00 \%)$ y comunicación $(5,00 \%)$. Asimismo, con porcentajes mínimos $(3,00 \%)$ se destacan otros estudios que se han encargado de analizar aspectos tales como internet, social media y web 2.0. Finalmente, también se registra un grupo pequeño de escritos $(2,00 \%)$ que se enfocan en aspectos como el big data, comercio electrónico, empresas virtuales, medios sociales digitales, m-learning, revolución digital y sociedad digital (ver Figura 4). 
Figura 4. Temáticas centrales de la producción científica

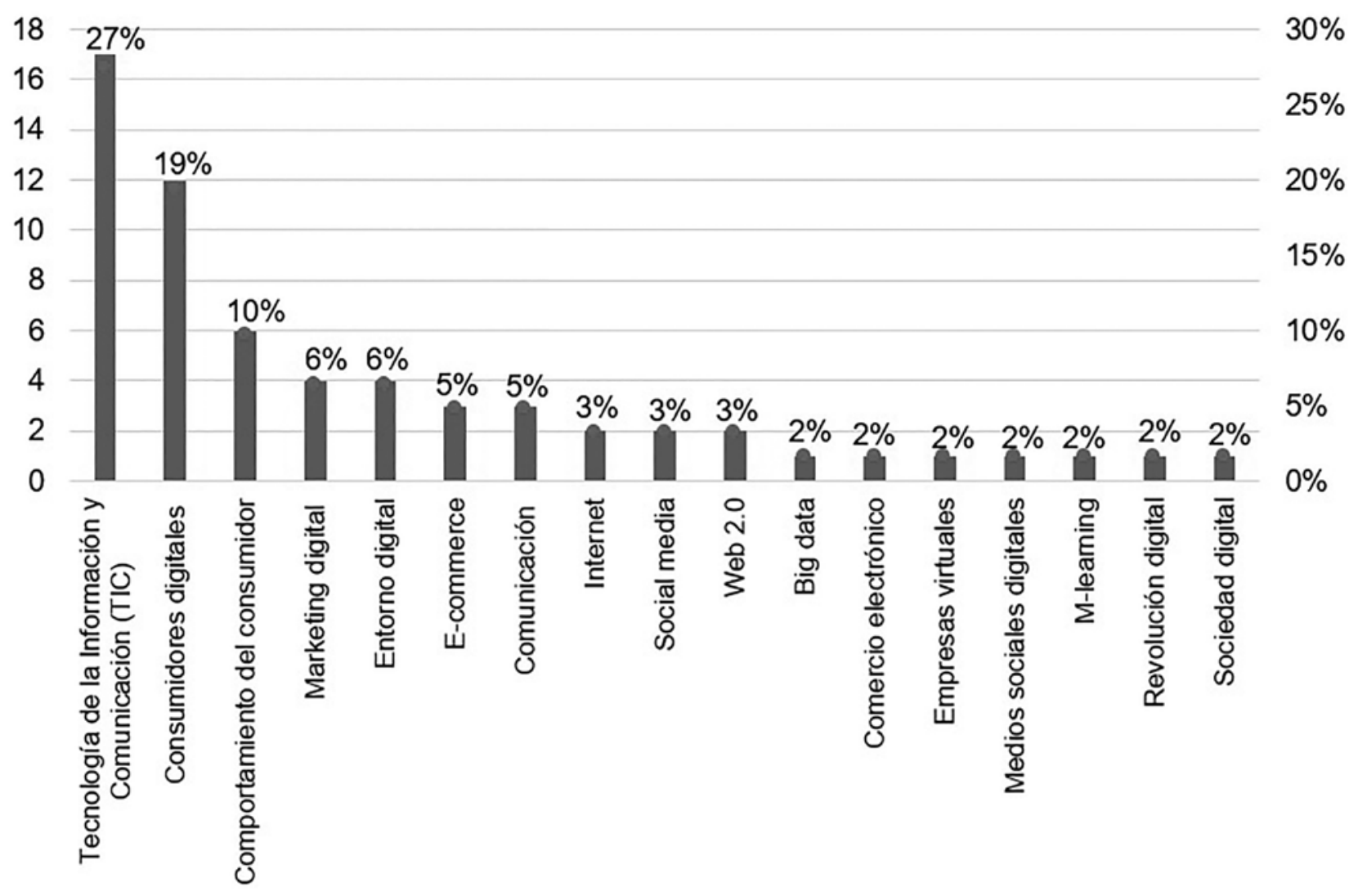

घ\# de trabajos $\bullet \%$ de trabajos

Fuente: Elaboración propia.

Como siguiente punto dentro de los resultados encontrados se presenta la información correspondiente al tipo de documento de donde se hizo la indagación correspondiente en torno al tema planteado, encontrando que los artículos en idioma español/inglés $(65,00 \%)$ son los que mayormente presentan estos trabajos. Muy distantes a este porcentaje se encuentran las tesis $(19,00 \%)$ y los libros $(16,00 \%)$.

\section{Figura 5. Tipo de documento utilizado}

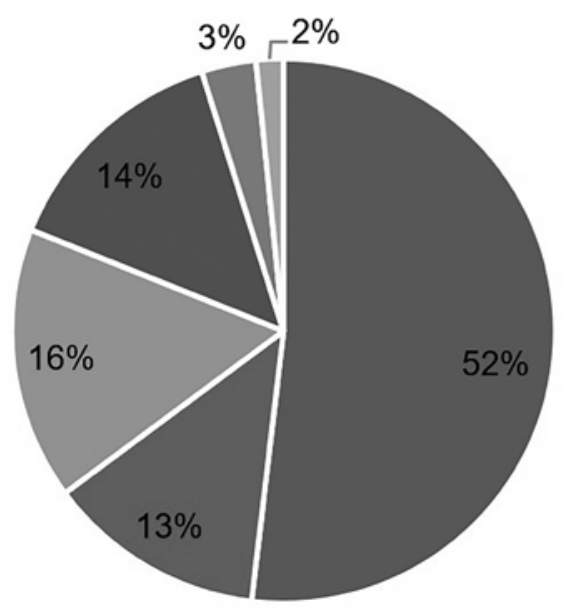

- Artículos de revista en español

- Artículos de revista en inglés

- Libros

- Tesis doctorales

- Tesis de posgrado

- Tesis de pregrado

Fuente: Elaboración propia. 
Adicional a lo ya presentado, resulta importante destacar la procedencia de los trabajos citados. Los mismos provienen en su gran mayoría de América Latina con el 53\%, siendo los lugares de origen países como Argentina, Chile, Colombia, Costa Rica, Cuba, Ecuador, México, Perú y Venezuela, el 37\% se desarrolla en Europa (Croacia, España, Francia y Portugal), el 7\% se desarrolla en América del Norte (Canadá y EEUU), y el 3\% restante se efectúa en Asia (China e India), recordando que para la búsqueda de información no existió restricción alguna en relación con el idioma empleado (Figura 6).

\section{Figura 6. Procedencia de los documentos}

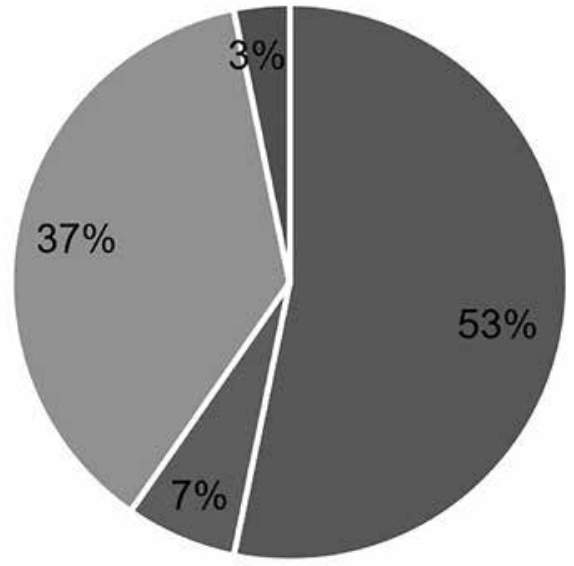

- América Latina

- América del Norte

- Europa

- Asia

Fuente: Elaboración propia.

A partir de lo expuesto en el epígrafe anterior, a continuación, se presentan los principales hallazgos en torno a los documentos analizados. De forma general, se pudo obtener información de 62 fuentes confiables y con una calidad de información para procesar la información a través de un proceso de lectura comprensiva y un posterior análisis por parte de los investigadores. Tal como se observa en la Figura 5, la información proviene de distintas fuentes bibliográficas de las cuales se detalló tanto categorías y subcategorías implícitas en las variables objeto de estudio (TIC y comportamiento del consumidor) que fueron utilizados en su debido tiempo en cada una de las investigaciones tomadas en consideración.

\section{Conclusiones y discusión}

La revisión de todos estos documentos muestra la importancia del tema planteado; dado que, los resultados obtenidos permiten visualizar los intereses de la comunidad científica en torno a estas líneas de investigación. En este sentido, según los registros obtenidos existe una alta producción científica especialmente entre los años 2014 y 2017 cuyos resultados han sido publicados en su mayoría en revistas indexadas.

A partir de ello, las grandes y profundas transformaciones generadas en el ámbito tecnológico han presentado varias repercusiones importantes en la opinión pública y en la vida social en general. Hoy en día, podría decirse que las distancias se han reducido enormemente, donde la tecnología ha dado paso a que los países por más distantes que se encuentren, gracias a las telecomunicaciones ahora sean más próximos, aprovechando de cierta forma el acceso que la tecnología ofrece (Bonilla et al., 2020). Entonces, a partir de este criterio, resulta comprensible que el desarrollo tecnológico experimentado especialmente durante los últimos años ha influido de sobremanera en el comportamiento de cada uno de los individuos, pues éstos actualmente se encuen- 
tran receptando una infinidad de información a cada instante, lo que repercute en su comportamiento (Molina et al., 2015). El alto tráfico de internautas en la web, permite la conformación de grandes comunidades virtuales, cuyos miembros comparten intereses parecidos. Por consiguiente, la interactividad que poseen las TIC rompe el esquema tradicional que maneja la comunicación, ante este panorama, los usuarios no únicamente consumen el contenido que ponen a su disposición los distintos medios, sino que también estos se encargan de compartir el contenido con otras personas (Álvarez \& Rodríguez, 2012).

Con la incursión del internet en el ámbito comercial, los procedimientos de compra han variado significativamente; dado que hoy en día, el cliente maneja mucha información en relación con la oferta de productos y/o servicios, y como un plus adicional, éste puede manejar diferentes alternativas para realizar la adquisición que la oferta local, nacional e inclusive internacional ofrece, ya que con la tecnología, las distancias ahora son un mito (Millat, 2011). Asimismo, su aplicación disminuye los problemas generados por la falta de información entre fabricantes y compradores; lo que no sucede con la incursión de la tecnología, pues la interacción es permanente, dotando al mercado la información como: disponibilidad de productos, características, precios, entre otros (García, 2014). De la misma forma ocurre con las TIC, que a lo largo de los años se han ido proliferando, los consumidores que actualmente hacen uso de la web para la compra de productos y/o servicios van en constante aumento, por consiguiente son pocas las personas que una vez que han utilizado este método lo llegan a rechazar (Martí et al., 2015).

Las TIC poco a poco se han convertido en un elemento clave en el crecimiento económico y en el desempeño de una empresa. Durante un tiempo, el impacto de las TIC pasó de la mera digitalización de los procesos manuales, que utilizan ordenadores a empresas completamente digitales (Berthon et al., 2012). Esto no es inusual, ya que los académicos han propuesto modelos en los que las nuevas tecnologías conducen a un largo período de innovación incremental, seguido de una interrupción radical por otra tecnología. La interrupción ocurre cuando los productos o servicios basados en nuevas tecnologías hacen que los productos o servicios existentes queden obsoletos (Kumar \& Thirumoorthi, 2019). La incursión de las TIC ha generado una transformación evidente en relación con el comportamiento del consumidor, es decir, el escenario comercial actual plantea que: "un nuevo consumidor que se encuentra más informado que antes, donde gracias a la presencia del internet y las redes sociales, se tiene acceso a una variedad de opciones, en cuanto se refiere a productos que se desea adquirir" (Millat, 2011, p. 628) de esta manera las opciones dadas pueden influir en su decisión de compra (Rinaldi \& Garmendia, 2015).

A partir de lo señalado, el incremento en relación con la utilización de las TIC hace que, tanto empresas como personas tomen la mayoría de decisiones dentro de un entorno digital; por cuanto en la actualidad es muy común ejecutar una serie de actividades, ya sea mediante páginas web o aplicaciones móviles, por ejemplo, desde la adquisición de ropa hasta la apertura de una cuenta bancaria; no obstante, a pesar de las enormes facilidades que brindan las TIC, es importante poner en consideración que en ocasiones, el usuario puede tomar decisiones erróneas debido al exceso de información disponible que puede ser fácilmente ignorada o no prestada atención (Weinmann et al., 2016).

El incremento en relación con el número de usuarios del e-commerce cada vez es más significativo, razón por la cual, el aumento de personas que requieren productos desde cualquier lugar del mundo, a cualquier hora del día y con una información completa de los productos a consumir va creciendo día tras día, lo que supone para la mayor parte de organizaciones un cambio de paradigma en cuanto a su gestión comer- 
cial (Salvi, 2014). Con el inicio de la era digital, tanto empresas como consumidores se hallan obligados a adaptarse ante este escenario; en este sentido, para el caso puntual de las empresas, es necesario que las mismas desarrollen actividades a corto plazo que permitan consolidar su desempeño en el mercado en el cual compiten. A partir de ello, una de las principales acciones a desarrollar se centra en planificar de forma más efectiva su accionar comercial, es decir, aprovechar al máximo la disponibilidad de estas nuevas tecnologías, ampliando en lo posible el abanico de productos que oferta y asegurando la fidelidad y confianza de sus clientes (Coloma \& Martín, 2014).

Los nuevos consumidores digitales han desarrollado nuevas rutinas dentro del proceso de compra, aspecto que se ha conseguido particularmente gracias al avance experimentado en relación con las nuevas aplicaciones disponibles acompañado del desarrollo comunicacional lo que ha permitido entender de alguna manera el comportamiento del nuevo consumidor, debido a que, éste se encuentra expuesto a un proceso de transformación de forma continua, abrupta y rápida (Bárcena et al., 2016). Este cambio constante se genera debido a que el usuario se encuentra conectado a la web las 24 horas al día y esto origina una perturbación en su forma de actuar, el cual se halla bombardeado de una infinidad de información y promociones que muchas de las veces termina influyendo en su decisión de compra (Alvear, 2017).

En la actualidad, las TIC han ganado un protagonismo significativo en diferentes áreas, dado que ha pasado a formar parte de la vida diaria de la mayor parte de seres humanos, donde su uso se encuentra inmerso en las distintas actividades que son desarrolladas día tras día. Adicional a ello, hoy en día, el consumidor está tomando decisiones de compra en una cultura digital participativa. El viejo comportamiento del consumidor ya no encaja dentro de esta era digital. Esto conlleva a una conclusión importante, donde la intervención de las TIC está inmersa en casi todas las fases de la toma de decisiones que realiza el consumidor. Sobre esta base, existen varios factores que influyen en este comportamiento y se vuelve muy importante para un vendedor comprender las preferencias de consumo, sus intenciones de compra en línea y sus comportamientos ante determinada marca, servicio, empresa o institución.

Los consumidores actuales se encuentran permanentemente conectados, presentan un alto poder de decisión, mayores alternativas de elección y requieren amplia información. Esta situación obliga a las distintas organizaciones a adaptarse ante este nuevo contexto a través del cambio de paradigma en relación con su gestión o el replanteamiento de acciones que permitan tener una interacción permanente con usuarios; es decir, dirigir sus esfuerzos a otorgar facilidades hacia los clientes quienes cada día que pasa son más exigentes pues como se ha visto a lo largo de este estudio presentan una cultura diferente en relación con la compra y adquisición de productos donde la tecnología tiene un rol fundamental para la consecución de dicha acción.

Finalmente, el nuevo escenario comercial plantea la sustitución de la moneda física por las transacciones electrónicas con el apoyo de tarjetas inteligentes, permitiendo a las compañías ofrecer un plus adicional en el proceso de comercialización que durante estos últimos años es visto de buena manera por los clientes quienes al momento de realizar sus compras buscan comodidad y sobre todo facilidades. Adicional a ello, se deja abierto, este estudio a la comunidad académica para que continúe investigando la variable comportamiento del consumidor en el contexto público, debido a que hoy en día, la irrupción de las nuevas plataformas digitales ha representado un cambio en el comportamiento social, tal es el punto que algunas instituciones públicas han tenido que adaptarse a esta revolución tecnológica obteniendo buenos resultados en los procesos de atención a la ciudadanía y una visión hacia el comportamiento del consumidor como una oportunidad de innovación. 


\section{Agradecimientos}

Un agradecimiento especial a la Universidad Tecnológica Indoamérica y la Editorial Queyám Cía. Ltda., por financiar el presente estudio.

\section{Referencias}

Álvarez-Tabares, O.J., \& Rodríguez-Guerra, E. (2012). El uso de la internet y su influencia en la comunicación familiar. Revista Trilogía, 7, 81-101. https://bit.ly/3soOYWC

Alvear, G. (2017). Consumidores turísticos digitales una alternativa para la sostenibilidad. Revista Universidad y Sociedad, 9(1), 161-164. https://bit.ly/3qWLbQ0

Apolo-Buenaño, D., Altamirano-Barriga, M.V., Vásconez-Cadena, V.E., \& Cevallos-Darquea, M.I. (2015). Usuarios, clientes y consumidores digitales: consideraciones para su abordaje desde el marketing y la comunicación corporativa. REDMARKA. Revista Digital de Marketing Aplicado, 1(14), 3-19. https://doi.org/https://doi.org/10.17979/redma.2015.01.014.4879

Arribas-Urrutia, A., Islas-Carmona, O., \& Gutiérrez-Cortés, F. (2019). De prosumidores a observadores: una tendencia emergente en Internet y en los jóvenes ecuatorianos. Resultados del Estudio del World Internet Project, Ecuador. Revista Latina de Comunicación Social, 74, 969-996. https://doi.org/10.4185/RLCS-2019-1367

Aucay-Piedra, E., \& Herrera-Torres, P. (2017). Nivel de uso de las redes sociales en el proceso de comunicación en las MIPES de Cuenca. Retos, 7(14), 81-98. https://doi.org/10.17163/ret.n14.2017.04

Bárcena, A., Prado, A., Cimoli, M., \& Pérez, R. (2016). La nueva revolución digital. De la internet del consumo a la internet de la producción. Comisión Económica para América Latina y el Caribe-CEPAL.

Barinas-Ubiñas, D. (2013). El impacto de las tecnologías de la información y de la comunicación en el derecho a la vida privada. Revista Electrónica de Ciencia Penal y Criminología, 15(9), 1-60. https://bit.ly/3dQxtuu

Barrientos-Felipa, P. (2017). Marketing + internet = e-commerce: oportunidades y desafíos. Revista Finanzas y Política Económica, 9(1), 41-56.

https://doi.org/http://dx.doi.org/10.14718/revfinanzpolitecon.2017.9.1.3

Barrio-Carrasco, J. (2017). La influencia de los medios sociales digitales en el consumo. La función prescriptiva de los medios sociales en la decisión de compra de bebidas refrescantes en España (tesis doctoral). Universidad Complutense de Madrid.

Barrios-Rubio, A. (2014). El comunicador en el entorno digital. Cuadernos.Info, 34, 165-181. https://doi.org/10.7764/cdi.34.519

Berthon, P., Pitt, L., Plangger, K., \& Shapiro, D. (2012). Marketing meets Web 2.0, social media, and creative consumers: Implications for international marketing strategy. Business Horizons, 55, 261-263. https://doi.org/10.1016/j.bushor.2012.01.007

Blanes-Peralta, C. (2017). Análisis del Marketing online para el desarrollo, comunicación e introducción a una empresa de servicios (tesis de pregrado). Universitat Politécnica de Valéncia.

Bonilla-Jurado, D.M., Lalaleo-Analuisa, F.R., Salinas-Morales, D.F., \& Masaquiza-Caiza, C.S. (2019). Perspectiva teórica de los proyectos de estimulación temprana: un acercamiento a partir del punto metaanalítico. Project, Design and Management, 1(1), 1-16. https://doi.org/10.35992/mlspdm.v1i1.173

Bonilla-Jurado D.M., Delgado-Salcedo, N., \& Fajardo-Aguilar, G.M. (2020). Branding, un elemento necesario del marketing estratégico en la Cámara de Comercio de Ambato. Revista Eruditus, 1(2), 9-25. https://doi.org/10.35290/re.v1n2.2020.278.

Burgos, E., Cerezo, J., Cortés, M., De La Cruz, X., Garolera, E., María, J., Godoy, G. J., Guardiola, J., Jimenez, R., Martínez-Priego, C., Monge, S., Pérez, J., Pino, I., Polo, J. L., Revuelta, J., Sánchez, J., \& Tejedor, R. (2009). Del 1.0 al 2.0: claves para entender el nuevo marketing. Bubok Publishing. 
Cáceres, G., Perea da Silva, T., Vázquez, M., \& Torres, M. (2017.) El impacto de los influencers de instagram en la decisión de compra de indumentaria de los jóvenes (tesis de pregrado). Universidad Argentina de la Empresa UADE.

Campos-Martínez, J.A. (2015). El uso de las TIC, dispositivos móviles y redes sociales en una aula de la educación secundaria obligatoria (tesis doctoral). Universidad de Granada.

Cano-Pita, G. (2018). Las TIC en las empresas: evolución de la tecnología y cambio estructural en las organizaciones. Dominio de las Ciencias, 4(1), 499-510. https://doi.org/http://dx.doi. org/10.23857/dom.cien.pocaip.2017.4.núm.1.enero.499-510

Capellan, N., Prince, A., Jolias, L., Berardi, M., Cristofani, E., Grobocopatel, G., López, J. O., De La Serna, J. M., Straface, F., Luna, D., Fontdevila, P., Ceria, S., Finquelievich, S., Dughera, L., Feldman, P., Piscitelli, A., Artopoulus, A., Bosch, M., Molina, M., Rotondo, S., Yoguel, G., \& Linskens, J. (2015). El impacto de las TIC en la economía y la sociedad. Cámara de Informática y Comunicaciones de la República de Argentina.

Coloma-Caturla, G., \& Martín-Antón, M.G. (2014). El impacto tecnológico en el comportamiento del consumidor. Una oportunidad para reactivar la economía española (tesis de posgrado). Pontificia Universidad Comillas.

Creswell, J. (2014). Research Design Qualitative, Quantitative, and Mixed Methodos approaches (4th Edi.). Sage Publications.

Cué-Brugueras, M., Díaz-Alonso, G., Díaz-Martínez, A.G., \& Valdés-Abreu, M. (2008). El artículo de revisión. Revista Cubana de Salud Pública, 34(4), 1-8. https://bit.ly/3aUMqtm

Dholakia, N., Zwick, D., \& Denegri-Knott, J. (2009). Technology, consumers, and marketing theory. The SAGE Handbook of Marketing Theory, February 2016, 1-512. https://doi.org/10.4135/9781446222454.n28

Díaz-Lazo, J., Pérez-Gutiérrez, A., \& Florido-Bacallao, R. (2011). Impacto de las tecnologías de la información y las comunicaciones (TIC) para disminuir la brecha digital en la sociedad actual. Cultivos Tropicales, 32(1), 82-90. https://bit.ly/3aWqiyK

Díaz-Mohedo, M.T., \& Vicente-Bújez, A. (2011). Los jóvenes como consumidores en la era digital. Revista Electrónica Interuniversitaria de Formación del Profesorado REIFOP, 14(2), 127-134. https://bit.ly/2NZgsmS

Encalada-Tenorio, G., Sandoya-Mayorga, L., Troya-Terranova, K., \& Camacho-Villota, J. (2019). El marketing digital en las empresas de Ecuador. Journal of Science and Research, 4, 1-10. https://doi.org/https://doi.org/10.5281/zenodo.3597830

Ferreirós-Bennett, P. (2016). Evolución del uso y aplicación del marketing digital, de las nuevas tecnologías y de las redes sociales en las empresas de la Provincia de Alicante entre los años 2011 y 2015 y su contribución a la consecución de sus objetivos empresariales y de marketing (tesis doctoral). Universidad CEU Cardenal Herrera, Valencia, España.

Ferrer, L. (2018). Comportamiento del consumidor 2.0: nuevas realidades en entornos digitales. Marketing Visionario, 7(1), 141-155. https://bit.ly/3pYY4aU

Gaile-Sarkane, E. (2009). Impact of technology adoption on consumer behaviour. Economics $\mathcal{E}$ Management, 14, 381-87. https://bit.ly/3r243gs

García de los Ríos, J.T. (2014). Comportamiento del consumidor en entornos 2.0 propuesta de un modelo de relaciones aplicado al sector turismo (tesis doctoral). Universitat de Valéncia.

Gil-Saura, I., Ruiz-Molina, M.E., \& Calderón García, H. (2009). La influencia de las TIC en la satisfacción del cliente en el comercio minorista. Cuadernos de Administración, 22(39), 59-73. https://bit.ly/3bIODat

Golovina, N. (2014). La comunicación masiva y el comportamiento del consumidor. Scientific E-Journal of Human Sciences, 28, 190-198. https://bit.ly/2O1UC2f

Guaña-Moya, E.J., Alvear-Escobar, A.G., \& Ortiz-Remache, K.J. (2015). Caracterización del consumidor digital ecuatoriano. Revista Publicando, 2(5), 226-242. https://bit.ly/37QTrcW

Guaña-Moya, E. J., Quinatoa-Arequipa, E., \& Pérez-Fabaraz, M.A. (2017). Tendencias del uso de las tecnologías y conducta del consumidor tecnológico. Ciencias Holguín, 23(2), 1-13. https://bit.ly/37MQrht 
Islas, O., Arribas, A., \& Gutiérrez, F. (2018). La contribución de Alvin Toffler al imaginario teórico y conceptual de la comunicación. Revista Latina de Comunicacion Social, 73, 648-661. https://doi. org/10.4185/RLCS-2018-1274

Kumar-Singh, A., \& Thirumoorthi, P. (2019). The impact of digital disruption technologies on customer preferences: The case of retail commerce. International Journal of Recent Technology and Engineering, 8(3), 1255-1261. https://doi.org/10.35940/ijrte.C4404.098319

Kutchera, J., García, H., \& Fernández, A. (2014). Exito su estrategia de marketing digital en 5 pasos. Grupo Editorial Patria.

Lagos-Reinoso, G.G., Mora-Barzola, K., Mejía-Caguana, D., Peláez-López, R., \& Peláez-López, J.C. (2018). M-learning, un camino hacia aprendizaje ubicuo en la educación superior del Ecuador. Revista Ibérica de Sistemas e Tecnologias Informação, 18(2), 47-60. https://bit.ly/3bEoc64

Linero-Bocanega, J.P., \& Botero-Cardona, L.F. (2020). Hábitos de consumo en plataformas e-commerce en adultos jóvenes de la ciudad de Bogotá. Revista Universidad Y Empresa, 22(38), 211-236.

https://doi.org/http://dx.doi.org/10.12804/revistas.urosario.edu.co/empresa/a.8131

López-Golán, M., Campos-Freire, F., López-López, P., \& Rivas-Echeverría, F. (2018). La comunicación en la nueva sociedad digital. Gráficas El Portatítulo.

Madrigal-Moreno, S., Madrigal-Moreno, F., \& Guerrero-Dávalos, C. (2015). Retos y oportunidades del social media en la organización contemporánea. European Scientific Journal, 11(23), 70-87. https://bit.ly/2ZRWe0S

Martí, F.P., Mañas-Alcón, E., \& Cuadrado Roura, J.R. (2015). El impacto de las TIC en las formas de consumo familiar. Economía Industrial, 360, 61-75. https://bit.ly/2PgwQ35

Matute, G., Cuervo, S., Salazar, S., \& Santos, B. (2012). Del consumidor convencional al consumidor digital. El caso de las tiendas por departamento. Esan Ediciones.

Mihajlović, I., Krželj, Z., \& Milić-Beran, I. (2014). Study of the impact of ICT on new trends in consumer behaviour in tourism when planning trips. Mathematics and Computers in Contemporary Science, 144-154. https://bit.ly/3uAPini

Millat, I. (2011). Flujo y comportamiento del consumidor en línea: un análisis empírico de las experiencias de consumo de productos formativos (tesis doctoral). Universitat Oberta de Catalunya.

Molina-Gómez, A.M., Roque-Roque, L., Garcés-Garcés, B.R., Rojas-Mesa, Y., Dulzaides- Iglesias, M.E., \& Selín-Ganén, M. (2015). El proceso de comunicación mediado por las tecnologías de la información. Ventajas y desventajas en diferentes esferas de la vida social. Medisur, 13(4), 481-493. https://bit.ly/3uCksdK

Organización para la Cooperación y el Desarrollo Económico OCDE (2019). Panorama del comercio electrónico políticas, tendencias y modelos de negocio. París, Francia: OCDE.

Ortiz-Morales, M.D., Joyanes-Aguilar, L., \& Giraldo-Marín, L.M. (2016). Los desafíos del marketing en la era del big data. Revista Escuela de Bibliotecología y Ciencias de la Información EBCI, 6(1), 1-29. http://dx.doi.org/10.15517/eci.v6i1.19005

Pelet, J.E. (2014). Consumer behavior in the mobile environment: An exploratory study of m-commerce and social media. International Journal of Technology and Human Interaction, 10(4), 36-48. https://doi.org/10.4018/ijthi.2014100103

Pelet, J., \& Papadopoulou, P. (2013). Handbook of research on user behavior in ubiquitous online environments. IGI Global.

Piñeros-Espinosa, R.A., \& Gómez-Santos, L.L. (2017). How can information and communication technologies (ICT) improve decisions of renewal of products and services and quest and selection of new suppliers? Espacios, 38(39), 28. https://bit.ly/37Q7I9z

Restrepo-Saldarriaga, N. (2011). Comunicación para el cambio social y nuevas tecnologías de la información y la comunicación. Revista Eticanet, 11, 158-178. https://bit.ly/3dNT4Uj

Rinaldi, E., \& Garmendia, M. (2015). El comportamiento de compra del consumidor de alojamiento turístico por internet. Revista Realidad, Tendencias y Desafíos en Turismo, 13, 26-50. https://bit.ly/3dPW4PZ 
Rivera, S.M., Castañeda, A., \& Rodríguez-García, J.E. (2012). Comportamiento de los consumidores en el uso de las tecnologías de la información y de la comunicación (TIC) (tesis de posgrado). Universidad EAN, Bogotá, Colombia.

Rivera-Sanclemente, M. del R. (2015). La evolución de las estrategias de marketing en el entorno digital: implicaciones jurídicas (tesis doctoral). Universidad Carlos III, Getafe.

Roman-Coy, D. (2016). Un análisis de las promociones de ventas al consumidor: los cambios que supone la introducción de los smartphones en esta táctica de marketing (tesis doctoral). Universitat Central de Catalunya, Barcelona.

Saavedra-García, M., \& Tapia-Sánchez, B. (2013). El uso de las tecnologías de información y comunicación TIC en las micro, pequeñas y medianas empresas (MIPyME) industriales mexicanas. Enlace, 10(1), 85-104. https://bit.ly/37Qvwu6

Salvi, F. (2014). Nuevo comportamiento del consumidor: la influencia del EWON (Electronic Word-Of-Mouth) en relación a la lealtad de los clientes en el sector hotelero (tesis doctoral). Universitat de les Illes Balears, Valencia, España.

Sánchez-Vázquez, J.F., Sánchez-Ordóñez, R., \& Jimbo-Días, J.S. (2019). "Happiness Management": Revisión de literatura científica en el marco de la felicidad en el trabajo. Retos, 9(18), 259-271. https://doi.org/10.17163/ret.n18.2019.05

Santos-Millán, I. (2019). El comportamiento del consumidor y las nuevas tendencias de consumo ante las TIC. Esic Market Economics and Business Journal, 50(3), 621-642. https://doi.org/10.7200/esicm.164.0503.4

Travaglini, M. (2016). La logística en las empresas virtuales (tesis doctoral). Universidad de Málaga.

Ureña-López, A., Carabias-Méndez, J. del A., \& San Segundo- Encinar, J.M. (2014). Tecnologías orientadas a la movilidad valoración y tendencias. Red.es y Fundación Vodafone.

Vega-Vega, J.A. (2015). Mercado digital único y consumidores. Revista de Estudios Económicos y Empresariales, 27, 87-116. https://bit.ly/37QMDfe

Vera-Salazar, P.H., Labarcés-Ballesta, C.A., \& Chacón-Velásquez, E.M. (2011). Influencia de las TIC en las organizaciones: Cambios y aparición de nuevas formas organizativas. Clío América, 5(10), 228-244. https://doi.org/https://doi.org/10.21676/23897848.420

Virgüez, J., Sánchez-Pineda, D.C., \& Rodríguez-Suancha, S.A. (2020). La comunicación de marketing en el comportamiento del consumidor para las organizaciones: revisión sistemática de literatura. Revista Boletín Redipe, 9(1), 174-183. https://doi.org/https://doi.org/10.36260/rbr.v9i1.906

Weinmann, M., Schneider, C., \& Vom Brocke, J. (2016). Digital Nudging. Business Eु Information Systems Engineering, 58(6), 433-436. https://bit.ly/3qYYcZv

Yasav, S. (2015). The impact of digital technology on consumer purchase behavior. EY Global Financial Services Institute, 3(3), 1-13. https://bit.ly/3uzzilb 